\title{
Interactions between model inclusions on closed lipid bilayer membranes
}

\author{
Timon Idema ${ }^{\mathrm{a}}$, Daniela J. Kraft ${ }^{\mathrm{b}}$ \\ ${ }^{a}$ email: t.idema@tudelft.nl \\ address: Department of Bionanoscience, Kavli Institute of Nanoscience, Delft University of Technology, \\ PO Box 5046, 2600 GA Delft, The Netherlands \\ ${ }^{b}$ email: kraft@physics.leidenuniv.nl \\ address: Soft Matter Physics, Huygens-Kamerlingh Onnes laboratory, Leiden University, PO Box 9504, \\ 2300 RA Leiden, The Netherlands
}

\begin{abstract}
Protein inclusions in the membranes of living cells interact via the deformations they impose on that membrane. Such membrane-mediated interactions lead to sorting and self-assembly of the inclusions, as well as to membrane remodelling, crucial for many biological processes. For the past decades, theory, numerical calculations and experiments have been employing simplified models for proteins to gain quantitative insights into their behaviour. Despite challenges arising from nonlinearities in the equations, the multiple length scales involved, and the non-additive nature of the interactions, recent progress now enables for the first time a direct comparison between theoretical and numerical predictions and experiments. We review the current knowledge on the biologically most relevant case, inclusions on lipid membranes with a closed surface, and discuss challenges and opportunities for further progress.
\end{abstract}

Keywords: membrane-mediated interactions, membrane-protein coupling, analytical models, coarse-grained models, self-assembly, DNA origami inclusions, colloidal model systems

\section{Introduction}

Living cells employ a wide range of proteins to carry out the various tasks needed to survive, function, and grow [1]. Many of these proteins are embedded in or attached to a cellular membrane. To perform a given task collaboratively on the membrane, proteins often need to get physically close to one another. Besides the electrostatic and van der Waals interactions, which are available to all proteins, membrane proteins can also interact through the membrane deformations they impose. These membranemediated interactions originate from the energetic costs for bending the membrane [2] and are considered to be of crucial importance for sorting and dynamically organizing proteins $[3,4]$.

Membrane-mediated interactions between proteins are difficult to study both in living cells and in re-constituted biomimetic systems, due to the small size of the proteins (well below the optical resolution limit) and the presence of other interaction terms. Although these interactions have been studied theoretically for 25 years, systems in which they can be directly measured have only been developed very recently [5, 6, 7]. These systems all 


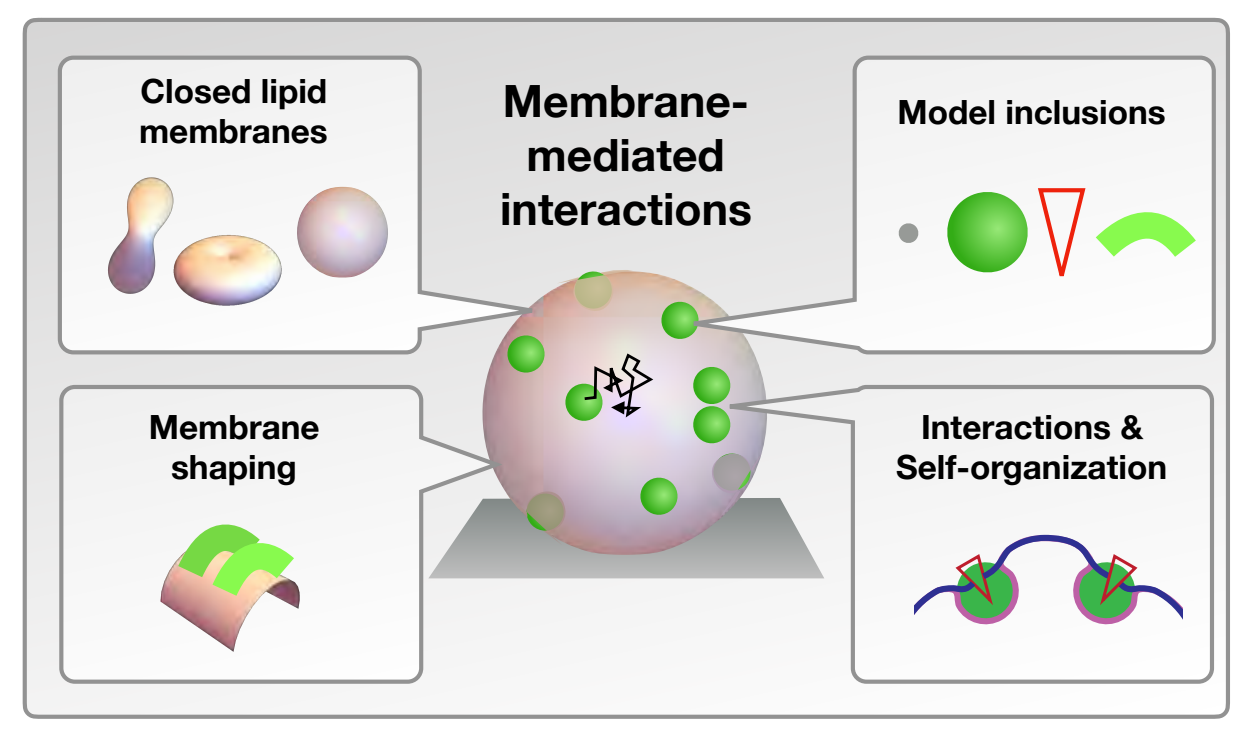

Figure 1: Model inclusions on closed lipid membranes: Proteins or other model membrane inclusions that adhere to and deform lipid membranes interact through these deformations. Although locally the deformation caused by the protein inclusions differs from the imposed shape of the colloidal model particles, at larger inter-particle distances the membrane shape is similar, resulting in similar interactions. We consider experiments, theory, and simulations of various model inclusions on closed lipid membranes, and discuss their interactions, self-organization, and their effect on the membrane shape.

use particles which are much larger than proteins, allowing for optical tracking, and either carefully eliminate or precisely quantify all other interactions. The resulting systems form a new class of soft materials which, apart from their role in quantifying the biologically relevant membrane-mediated interactions, will have applications in developing hybrid materials and as models for understanding uptake and retention of nano- and micrometresized particles by organisms.

Figure 1 shows our system of interest: model inclusions either adhered to or included in a closed membrane, locally deforming it and thus interacting with other included or adhered particles. The particles may either sit on the same or the opposite side of the membrane and come in various shapes. In the simplest case of spherical particles or conical inclusions, the imposed deformations will be isotropic, but even isotropic particles may self-assemble into complexes that together deform the membrane in an anisotropic manner.

The purpose of this review is to give an overview of the advances made in describing and quantifying the interactions between membrane-inclusions on flexible and fluid lipid membranes. We focus on closed lipid membranes as they are the most biologically relevant case and only consider membrane-mediated interactions induced by deformations of the membrane. The structure of this article is as follows: we first describe the available theoretical frameworks on flat membranes and review simulation methods suited for numerically investigating membrane-mediated interactions. Then, we review the quantitative measurements, observed inclusion patterns and membrane deformations in experimental model systems. Finally, we discuss the progress that has been made in analytical approaches and simulations to elucidate how multiple isotropic and aniostropic inclusions interact on closed lipid membranes. 


\section{Background}

\subsection{Theoretical work on flat membranes}

Since a bilayer membrane is only $4-5 \mathrm{~nm}$ thick but up to hundreds of microns wide, it is not unreasonable to use a theoretical description in which the membrane is coarsegrained to an infinite thin sheet, possibly with a term accounting for the difference in composition of the two leaflets. This approximation not only works for adhered particles that are significantly larger than the membrane thickness, but can also be applied to protein inclusions modelled as point particles that locally impose curvature. Since lipid membranes are in-plane fluids but resist out-of-plane bending, curving the membrane carries an elastic penalty. The widely used Canham-Helfrich free energy accounts for this elastic property by expanding membrane deformations up to second order in the curvature. Its free energy functional is given by $[8,9]$ :

$$
E_{\mathrm{CH}}=\int\left[\frac{\kappa}{2}\left(2 H-C_{0}\right)^{2}+\bar{\kappa} K+\sigma\right] \mathrm{d} A,
$$

where $H$ and $K$ are the membrane's mean and Gaussian curvature, $C_{0}$ is any spontaneous curvature due to bilayer asymmetry, $\kappa$ the bending modulus, $\bar{\kappa}$ the Gaussian modulus, and $\sigma$ a Lagrange multiplier that keeps the membrane area fixed, or, alternatively, the membrane's surface tension penalizing changes in area. As the Gaussian curvature integrates to a topological constant over a closed surface, it is typically left out of consideration. Although the Canham-Helfrich and various derived models of the membrane have been studied for decades, a general expression for a membrane shape that minimizes it has not yet been found, due to the nonlinear nature of the corresponding shape equation. There are many approximate results for the linearised case and numerical ones for the full model, accounting for variations in membrane composition and boundary conditions imposed by the inclusions we discuss here.

The first study of membrane-mediated interactions based on the Canham-Helfrich free energy was done by Goulian, Bruinsma and Pincus [2] in tension-free and flat membranes. They found an interaction potential $F \sim \kappa\left(\alpha_{1}^{2}+\alpha_{2}^{2}\right) / r^{4}$, where $\kappa$ is the membrane's bending modulus, $r$ the distance between the inclusions, and $\alpha_{1}$ and $\alpha_{2}$ the angles they impose at their edges (the opening angles of the cones shown in figure 1). Note that the result of Goulian et al. predicts that the interaction between like and opposite inclusions is identical, as it scales with the sum of the squares of the imposed angles,and always repulsive. Goulian et al. also showed that thermal fluctuations would lead to an additional effect, of order $1 / r^{4}$. Park and Lubensky [10] refined this calculation, showing that thermal fluctuations would lead to an order $1 / r^{4}$ effect for inclusions with up-down symmetry, but of order $1 / r^{2}$ for ones that break this symmetry. They also accounted for the possibility that the deformations imposed by the inclusions are not isotropic. Kim, Neu and Oster [11] showed that despite the pairwise repulsions, manybody interactions can play an important role. Later, Yolcu et al. [12] showed that higher order multibody terms beyond three-body terms need to be taken into account as well. The result of Goulian et al. was extended to membranes with tension by Weikl, Kozlov and Helfrich [13], for which case there is an additional leading term $2 \pi \kappa \alpha_{1} \alpha_{2}(\xi a)^{2} K_{0}(\xi r)$, where $\xi=\sqrt{\sigma / \kappa}$ is the inverse of the characteristic length scale, $\sigma$ the tension, $a$ the radius of the (conical) inclusions, and $\alpha_{i}$ their opening angle. Due to this additional 
term, the interaction becomes attractive at large separations for opposite inclusions on a membrane with tension, while remaining repulsive for identical conical ones and at short distances.

In 2003, Evans, Turner and Sens [14] studied a closely related system, where inclusions are assumed to exert a force rather than impose a contact angle on the flat membrane. They found repulsive interactions for isotropic inclusions and attractive interactions for anisotropic ones; they also derived the Green's function for the linearised shape equation of the membrane with tension. Meanwhile, Dommersnes and Fournier [15] had developed an analytical solution for the energy of a system with many inclusions in the limit that they can be described as point-like, locally imposing the curvature rather than a contact angle at the rim of an extended particle. They predicted that for tension-free membranes, many-body interactions between anisotropic (saddle-like) inclusions would result in the formation of ring-, line- and egg-carton like patterns. Weitz and Destainville [16] added tension to the model of Dommersnes and Fournier, showing that they could re-derive the interaction potential of Weikl et al. using point-like particles, and that many of these particles could spontaneously aggregate. Notably, Guven, Huber and Valencia [17] studied the interaction of helical inclusions, which exhibit long-range attraction and shortrange repulsion, and can describe the 'Terasaki spiral ramps' or 'parking garage shapes' found in the endoplasmic reticulum of the cell [18].

An alternative approach to describe membrane-mediated interactions is through the membrane's stress tensor, as first described by Capovilla and Guven [19]. Müller, Deserno and Guven [20] used this method to derive an exact expression for the force between two identical inclusions. The advantage of this approach is that it allows for arbitrarily large deformations. Unfortunately, the expression for the force cannot be evaluated in closed form, though Müller et al. could infer the sign of the force for two infinite cylinders. Yet another method is to employ an effective field theory to calculate interactions as developed by Yolcu and Deserno [21]. This field-theoretical method again only applies to small deformations, but can be used to systematically obtain pairwise as well as threeand four-body potentials [12].

In summary, on flat membranes with tension identical isotropic inclusions are predicted to repel, while opposite as well as anisotropic particles are predicted to attract each other at long distances and repel at short separations. However, the various methods described above have two important drawbacks. First, they are almost all restricted to small deformations, and second, biological membranes are closed and hence curved surfaces. Similarly, experimental lipid membrane model systems need to be free standing to allow inclusions to impose a deformation on the membrane and thus often giant unilamellar vesicles (GUVs) are employed. The membrane-inherent curvature has a significant effect on the interactions and assembly pattern of membrane inclusions as we will show in the subsequent sections. Although some analytical work has been done on closed and curved membranes (section 4.1), large deformations remain out of reach due to inherent nonlinearities. Fortunately, simulations can be carried out in that regime, and used to bridge the gap between experiments and analytical work.

\subsection{Simulation methods}

All-atomistic simulations cannot (yet) reach the time and length scales necessary to study membrane-mediated interactions and therefore simulations are typically carried out 
at the coarse-grained molecular and coarse-grained near-continuum level. Coarse-grained but still molecular simulations were done by Reynwar et al. [22], who used a model in which lipids are described by three spheres (one hydrophilic and two hydrophobic), forming a flat fluid bilayer spanning the simulation box. Adsorbed particles were constructed from beads of the same size as the lipids, with an attractive interaction between some of the particle beads and the hydrophilic beads in the lipids. In contrast to most analytical models, these simulations predict that inclusions attract up to very short distances, and collectively can induce the membrane to undergo vesiculation or even budding. A more detailed but still coarse-grained description is employed in the MARTINI model, in which a single bead represents four heavy atoms and their associated hydrogens [23, 24]. The MARTINI model is widely used for studying the dynamics of the lipid bilayer and its direct interaction with transmembrane proteins, but remains too detailed for the scales of membrane-mediated interactions.

To describe membranes at micrometre rather than nanometre length scales, they need to be coarse-grained further, to a level where beads no longer represent atoms or even molecules, but patches of a membrane. Such a description can therefore also be considered a discretised version of the continuum model of Canham and Helfrich. The integral over the mean curvature can be approximated by triangulating the surface, and calculating the projection of the normal of neighbouring triangles onto each other. One can account for the in-plane fluid nature of the membrane by allowing edges between neighbouring triangles to flip. The Metropolis Monte-Carlo method can then be used to find minimalenergy configurations of the membrane with adhering particles [25, 26, 27]; these methods were reviewed by Sreeja et al. [28]. Alternatively, one can use finite-element methods [29] or relaxation methods [30] to find membrane shapes minimizing the curvature energy.

The various simulation methods can of course complement each other, and have all lead to new predictions and insights. For our purposes, the rough coarse-grained approach holds the most promise, as it can simulate the entire system at relevant timescales, and still connect to both the experiments and the analytical work, as discussed in the following sections. With the advance of computing power, the coarse-grained mesh can become more refined, and connect to the lipid-based or perhaps even all-atomistic models. Already with current technology explicit predictions can be made about the patterns that many inclusions can make, and the step to designing these inclusion patterns can now be taken. A key development of the last couple of years that allows for this next step is the experimental quantification of the interaction potential between membrane inclusions.

\section{Experimental measurements of membrane-mediated interactions on GUVs}

The reason for the startling lack of quantitative knowledge of membrane-mediated interactions between proteins is that experimental studies of such interactions between individual proteins are difficult.

One challenge lies in gathering data on their distance dependant interaction potential: proteins are too small to be resolved by in situ techniques such as light, fluorescence, confocal, and superresolution microscopy upon approach. Fluorescent markers can be employed for studying the behaviour of large numbers of proteins, but are not suited for measuring membrane-mediated interactions between individual proteins because of their bulky nature. Transmission electron microscopy allows atomic-level information 
of proteins and their aggregates on membranes but is restricted to thin, solid samples precluding the investigation of their dynamics. This may be resolved by the developing technique of liquid cell electron microscopy, which allows imaging of thin liquid specimens [31, 32]. Small angle X-ray scattering (SAXS) measurements have recently been used to extract the interaction energy, for example between alamethicin pores [33] and gramicidin channels [34]. However, protein interactions are usually not limited to membrane deformations only but include electrostatic, hydrophobic/hydrophilic and van der Waals interactions, making it challenging to single out the contribution stemming from the membrane deformation. The typically anisotropic protein shape which may furthermore be affected by interaction with the membrane or other proteins further complicates quantification and direct comparison with theoretical models. Finally, protein interactions with the membrane on the molecular level may lead to additional effects such as local sorting of lipids that go beyond bending and tension mediated interactions and cannot be captured by coarse-grained or elastic-continuum models of the membrane.

Therefore, proteins are not the first choice for testing predictions and quantitatively studying membrane mediated interactions. Better suited are model systems that mimic membrane inclusions while overcoming some of the challenges inherent to proteins. The first experiments with model inclusions on lipid membranes were done by Koltover et al. [35]. They observed that membrane deforming objects, be they colloidal particles or DNA-lipid aggregates, strongly deform flaccid membranes and attract over a distance of roughly the diameter of the inclusion. They furthermore noticed that spherical particles were predominantly found in regions of negative curvature and observed collective arrangements into close packed hexagonal clusters or rings around the waist of dumbbell shaped vesicles (figure 2d). After these exciting initial observations, no further quantification of the process has been reported for almost two decades until recently. In this section, we will discuss the progress that has been made with model systems made by DNA-origami constructs and colloidal particles in the past years.

\subsection{Model inclusions made from DNA-origami}

Model inclusions based on DNA origami profit from the versatility of this technique in creating virtually any shape and straightforwardly designing the position of interaction sites and their strength. Key to anchoring DNA origami constructs to lipid membranes is the presence of lipophilic moieties, such as cholesterol or tocopherol, whose number and chemical nature determines the adhesion energy. This emerging technique has recently for the first time been employed to study membrane-mediated interactions, albeit not yet quantitatively, possibly because the origami constructs are below the resolution limit of conventional microscopy techniques.

Flat, rectangular origami blocks that bind to other blocks can deform GUVs [36] and SUVs [37] by imposing a planar deformation, that may even destroy the vesicle [37] (figure 2a). The assembly of the endosomal sorting complexes required for transport (ESCRT) subunit Snf7 inspired Grome et al. to investigate the effect of DNA origami curls on lipid vesicles. Dense coverage and polymerization of GUV and LUV adhered DNA origami curls into springs was shown to induce the formation of tubes or even complete transformation of LUVs into a spring covered tubes (figure $2 \mathrm{~b}$ ). The resultant tubes were densely covered with the DNA constructs oriented perpendicular to the tube [38]. To mimic BAR domains Franquelim et al. created $100 \mathrm{~nm}$ linear and curved rods with dif- 
a)

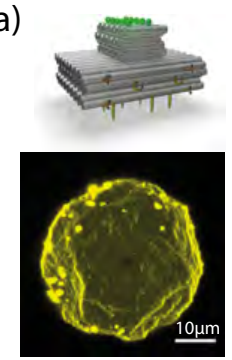

f)

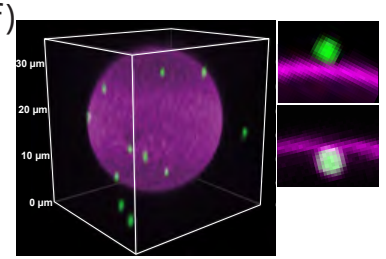

b)
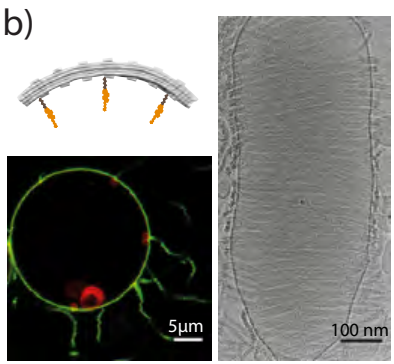

c)
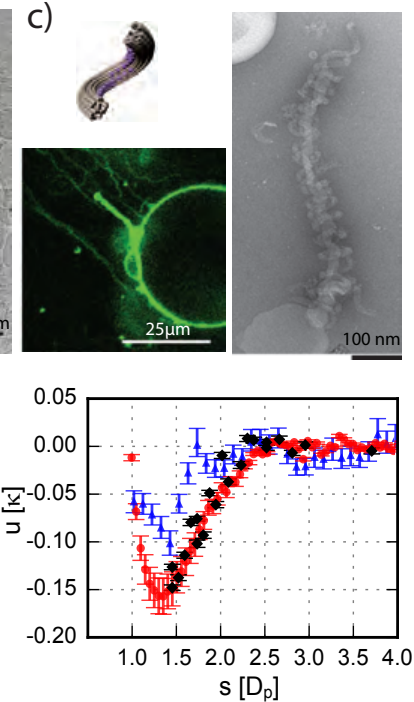

d)

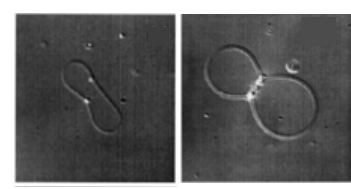

e)

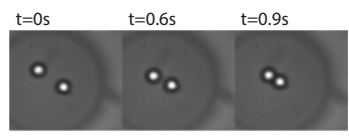

g)

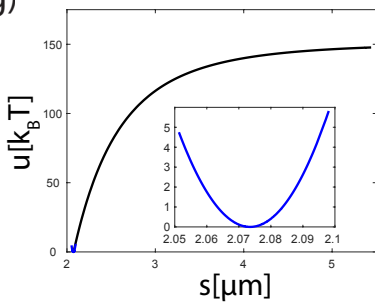

Figure 2: Examples of experimentally found inclusion interactions on lipid vesicles a) Interacting DNA origami blocks deform GUVs, from Czogalla et al. [36] reprinted with permission of John Wiley \& Sons, Inc. b) Concave DNA-origami rods induce outward vesicle tubulation in deflated vesicles. TEM (right) shows a dense packing of the rods on tubules, from Franquelim et al. [39] (CC BY 4.0). c) Self-assembly of DNA curls triggers the spontaneous formation of spring-covered tubules from lipid vesicle. TEM micrograph shows how the spring surrounds the strongly deformed vesicle, from Grome et al. [38] reprinted with permission of John Wiley \& Sons, Inc. (d) Colloidal particles on GUVs preferably locate in regions of negative curvature, reprinted with permission from Koltover et al. [35], copyright (1999) by the American Physical Society. e-g) Spherical colloidal particles that deform GUVs attract each other. e) Light microscopy sequence of a pair of colloids, reprinted with permission from Sarfati and Dufresne [6], copyright (2016) by the American Physical Society. f) 3D reconstruction of confocal microscopy stack showing colloids (green) on a GUV (magenta), with insets showing attached (top) and wrapped (bottom) states and image from a simulation at particle separation $s=1.5 D_{p}$; Interaction potential between two spherical particles measured in the experimental system for tense (blue triangles) and floppy (red dots) vesicles and in simulations (black diamonds), from van der Wel et al. [5] (CC BY 4.0). g) Experimental measurements of the interaction potential between two spherical particles in system e), inset shows the near field potential, from Sarfati and Dufresne [6].

ferent radii of curvature made from DNA origami [39]. By placing the membrane anchors on the positively or negatively curved region of the rods, deformations with a different curvature could be imposed onto lipid vesicles. They found that linear scaffolds had no strong effect on the GUV shape, whereas concave scaffolds could trigger outward tube formation and convex scaffolds induced invagination type deformations above a critical concentration. Highly curved constructs did not induce tubulation due to a competition between the adsorption energy and bending energy. Outward tubes were found to be densely covered by DNA nanostructures perpendicularly aligned along the rod axis, see figure $2 \mathrm{~b}$.

The freedom to design DNA constructs of virtually any shape and control over number, position and type of membrane anchors opens the door to create and study model inclusions that mimic proteins involved in membrane shaping. This promising research avenue has just been begun to be explored and already provided fascinating new insights into how membrane-deforming proteins collectively assemble and alter membrane shapes. 


\subsection{Colloidal particles as model inclusions}

A promising type of model membrane inclusions are colloidal particles. Their diameter can be chosen large enough to allow visualization by light microscopy techniques and small enough to still undergo thermal motion, typically between a few hundred nanometre to a few micrometre. This allows quantitative detection of their trajectories using image analysis and thereby inference of their interactions. The adhesion of colloidal particles to lipid membranes can be achieved in various ways: (1) by non-specific interactions, such as electrostatic interactions between charged beads and lipids with an oppositely charged headgroup, hydrophobic interactions, or van der Waals interactions [7, 40,35, 41, 42]; and (2) by specific receptor-ligand based interactions, e.g. between streptavidin coated beads and GUVs doped with biotinilated lipids [43, 35, 5, 44].

After adhesion to the membrane, the particles become (partially) wrapped by the membrane depending on their interaction with the membrane, their shape and orientation with respect to the membrane given a sufficiently low membrane tension $[45,46]$. For colloidal particles, the short interaction range with respect to the particle diameter leads to a very sharp wrapping transition with increased adhesion strength and high degrees of wrapping $[5,40,47]$.

The transition from the adsorbed to wrapped state was found to occur in a few milliseconds and sometimes has been observed to be followed by a slower "ingestion" process that lead to a further transfer of the particle towards the vesicle center and concomitant decrease of the vesicle size [40]. Fery et al. followed the membrane coverage of particles in contact with a tensionless GUVs and showed that the wrapped state can be achieved even if the particles are not allowed to move towards the vesicle center [41]. More recent confocal microscopy experiments on spherical colloids have furthermore confirmed that the adhesion strength, which can be tuned through the receptor surface density, determines whether the particles are fully wrapped or just adhered [5] (figure 2e). Particle coverage by lipid membrane requires the availability of excess membrane area and conversely leads to a increase in membrane tension $[5,41]$.

Particles that are at least partially wrapped by the membrane will interact through their membrane deformation and can thus be used as a model system to investigate and quantitatively measure membrane-mediated interactions. Upon wrapping, rigid particles impose their shape onto the membrane, while soft particles can be deformed themselves to minimize the overall free energy and may feature temperature-dependent interactions [42]. Thus, quantitative measurements of membrane-mediated interactions on soft particles are more involved and hence only rigid particles have been used to date. To be able to single out the interactions due to the membrane deformation it is important that all other interactions between colloidal particles are either suppressed or known. Also, because particles that induce deformations sense and respond to membrane curvature [7], quantitative measurements of membrane-mediated interactions need to take the overall membrane shape into account.

After the initial qualitative observations of attractive interactions between model inclusions by Koltover et al. in 1999, the quantification of these interactions was independently tackled by two groups in 2016. Experiments by Sarfati and Dufresne confirmed that streptavidin-coated polystyrene beads - although stable in solution - attract each other on tense GUVs over distances of roughly a bead diameter [6]. On flaccid GUVs, no interaction was observed. In the bound state, the particles fluctuated around an equi- 
librium separation close to the particle diameter. They extracted the force profiles and interaction energies using a maximum likelihood analysis. The extracted interactions were found to vary greatly and reported to be as large as $150 k_{\mathrm{B}} T$, see (figure $2 \mathrm{e}$ and g). The variation has been attributed to a non-uniform degree of membrane wrapping and dependence on the (uncontrolled) membrane tension. The authors hypothesize that multipolar tension-mediated capillary interactions due to roughness of the contact line between membrane and particles are the origin for the observed attraction.

To single out membrane-mediated interactions, van der Wel et al. designed their colloidal model system to exclude effects from gravity and suppress van der Waals and electrostatic interactions [5, 44]. Using confocal microscopy they observed that membraneadhered particles were either not wrapped or fully wrapped, which could be controlled by particle-membrane adhesion strength and membrane tension [5], see figure $2 \mathrm{f}$. They extracted the interaction potential using a transition probability matrix approach and found that membrane-attached but not wrapped particles only diffused on the membrane without any interactions [5, 44]. Two membrane-wrapped particles on the other hand attracted each other with about $-3 k_{\mathrm{B}} T$ energy over a range of about one bead diameter on floppy vesicles $(\sigma>10 \mathrm{nN} / \mathrm{m})$, and slightly less on tense vesicles $(\sigma>1 \mu \mathrm{N} / \mathrm{m})[5]$ (figure 2f). Monte-Carlo computer simulations quantitatively confirmed the measured two-particle interaction (figure $2 \mathrm{f}$ ). The authors conclude that the observed attraction is due to a decrease in the bending energy of the membrane upon approach of the particles [5]. Pairs of wrapped and non-wrapped particles can assemble into a dimer state and subsequently wrap both particles into a tubular state. Whenever mixtures of wrapped and non-wrapped particles were present on the membrane, irreversible aggregation ensued in the long term [44].

The above discussed experiments have consistently found attractive interactions that extend over roughly a particle diameter between spherical colloidal particles that deform spherical lipid membranes. An open question remains as to the relative contributions of membrane bending and membrane tension to the measured attraction, which could be resolved by using a micropipette aspiration setup to control the membrane tension. Further control over the degree of wrapping and the wrapping angle, for example by employing Janus particles that only partially adhere to the lipid membrane [7], will be an exciting avenue to quantitatively test predictions on the two-particle interactions from theory and simulations [48]. Already, Janus particles have been observed to also attract on GUVs and pairs orient themselves in curvature gradients [7]. Colloidal model inclusions with an anisotropic shape would open the door to dynamically measure non-spherically symmetric interactions, which are highly relevant in biology, for example for proteins with BAR domains. Recent experiments on semiflexible rod-like fd virus particles showed a rich assembly into linear chains, collapsed globules, and chain-like aggregates on membranes depending on the adhesion strength [49]. A quantitative measurement of the orientationdependant membrane-mediated interaction potential of these particles has not been done yet but should be feasible. Finally, experiments on colloid-lipid membrane model systems provide an excellent means to study the complex and non-additive interactions between multiple particles and the states they assemble into. 
a)

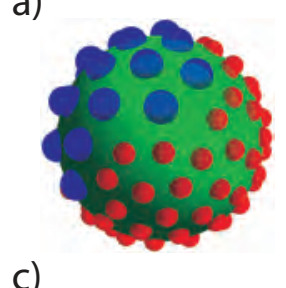

c)

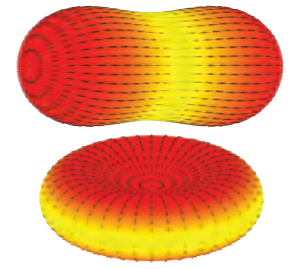

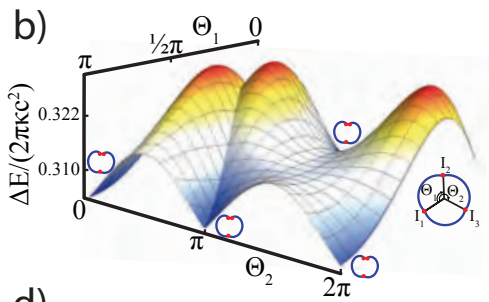

d)

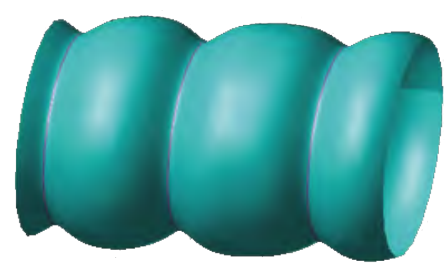

Figure 3: Examples of inclusion arrangements on curved and closed membranes from recent analytical work. a) Mixtures of inclusions on a spherical vesicle are sorted according to inclusions size, reprinted with permission from Idema et al. [50], copyright (2010) by the American Physical Society. b) On cylindrical membranes, two long rods position themselves on opposite sides, but adding a third rod results in a minimum-energy state in which two rods coalesce, reprinted with permission from Vahid and Idema [51], copyright (2016) by the American Physical Society. c) Flow fields on deformed vesicles, showing how inclusions adsorbed from the outside or inside migrate towards regions of low curvature (top) or high curvature (bottom), respectively. Reproduced from Agudo-Canalejo and Lipowsky [52] published by The Royal Society of Chemistry (CC BY 3.0). d) Ring-like inclusions on tubular membranes can coalesce under specific conditions, from Shlomovitz and Gov [53].

\section{Interactions between inclusions on curved and closed membranes}

\subsection{Analytical work}

As detailed in section 2.1, analytical work on membrane-mediated interactions was initially done on infinite membranes that are asymptotically flat. Cellular membranes, however, are usually curved and closed surfaces, as are the membranes in many biomimetic experimental settings. In general, inclusions behave differently on curved and closed membranes than they do on flat ones.

In 1998, Dommersnes, Fournier and Galatola [54] showed that for small separations on a spherical membrane, two identical conical inclusions would repel as $1 / \theta^{4}$, like the result of Goulian et al. [2] on tensionless flat membranes. For larger angles however, the globally curved and closed nature of the spherical topology becomes significant, resulting in a repulsion which is stronger than for the flat case, and an eventual equilibrium with the inclusions on opposite poles. Idema et al. [50] showed that such interactions could lead to spontaneous sorting of inclusions of different size (figure 3a). Adhered colloids, on the other hand, impose a curvature that is opposite to the global curvature of the sphere, and therefore will attract each other [5]. Moreover, Agudo-Canalejo and Lipowsky recently showed that such adhered colloids can detect local curvature differences on a stretched sphere, predicting that they will migrate to low-curvature regions when adsorbed from the outside of a vesicle and to high-curvature regions when adsorbed from the inside [52] (figure 3c).

On tubular membranes, as often found inside cells, Vahid and Idema [51] analytically studied point-like inclusions and predicted the formation of rings of inclusions and the arrangement of rings and rods, see figure $3 \mathrm{~b}$. The interactions and dynamics of rings on 
tubes have earlier been investigated by Shlomovitz and Gov [53], who identified conditions under which they could coalesce, see (figure 3d). Vahid and Idema found similar results for rings, and showed that while identical long rods will sit on opposite sides of the tube, adding a third identical rod will not result in a configuration with equally spaced inclusions, but cause two of them to coalesce. This demonstrates both the non-additive behaviour of the inclusions and the strong impact of the closed geometry. Moreover, they predicted the formation of rings of isotropic particles, later confirmed in simulations [55] (see figure $4 \mathrm{~d}$ ).

The amount of analytical results available for closed membranes remains small to date, predominantly due to the fact that while the relevant equations are relatively easy to write down, they are exceedingly difficult to solve. Fortunately, simulations have in recent years been able to reproduce both the available analytical and experimental results, and are leading the way in revealing possible emerging patterns in a broad range of biologically and technologically relevant settings.

\subsection{Simulation work on colloids on closed vesicles}

In 2012, two groups independently reported simulation results that showed the spontaneous aggregation of colloids adsorbed to GUVs [26, 27, 61]. These results at first glance seem to contradict the analytical one of Dommersnes et al. [54], but the setting is slightly different: where the analytical work was on inclusions that impose a given tangent at their edge, the simulations are of colloids that are partly wrapped by the membrane, and can share their wrapped region, leading to a global energy minimum in which they are close together. Both simulation groups also showed that multiple colloids together can induce the formation of membrane tubes on the vesicle, due to a larger contact area between colloid and membrane. Follow-up simulations based on the method of Sarić and Cacciuto [26, 61] by Vahid et al. allowed for the first time direct comparison between simulation and experimental data on the interaction of two adsorbed colloids, as reported in [5]. Very recently, Bahrami and Weikl also published follow-up results to their 2012 work, in which they show that partially wrapped colloids (figure 4e) will attract when adsorbed to the outside of a vesicle, but repel when adsorbed to the inside [59].

On non-spherical vesicles, colloids inducing deformations interact with the curvature of the vesicle. Multiple adsorbed colloids will arrange in structures that seek out the region of lowest curvature [58], in accordance with the analytical work of Agudo-Canalejo and Lipowsky [52]. In particular, colloids adsorbed to prolate ellipsoidal vesicles will spontaneously form a ring around the vesicle's midplane [58] (figure 4c).

\subsection{Anisotropic inclusions on curved and closed membranes}

As already noted by Park and Lubensky in 1996 [10], anisotropic inclusions may behave differently than their isotropic counterparts. Anisotropy in the imposed deformation of the membrane can originate from the shape of the inclusion itself, for example in the banana-shape of proteins with a BAR domain (reviewed in Simunovic et al. [62]). Alternatively, anisotropy can arise from the combined deformation of two or more linearly arranged isotropic inclusions, leading to effective multi-body interactions as studied by Kim et al. [11] and Dommersnes and Fournier [15]. Where isotropic inclusions on the same side of a flat or spherical membrane are predicted to repel, anisotropic ones are generally predicted to attract and form aggregates. 
a)

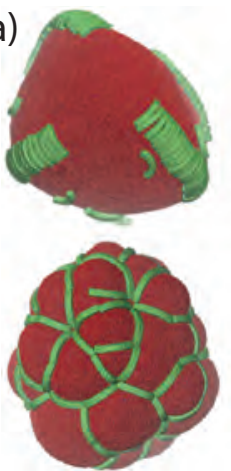

e)

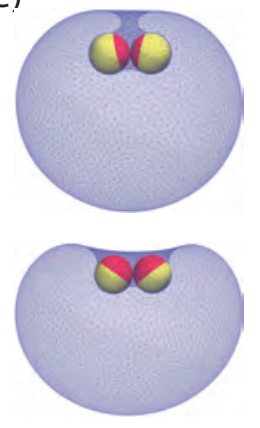

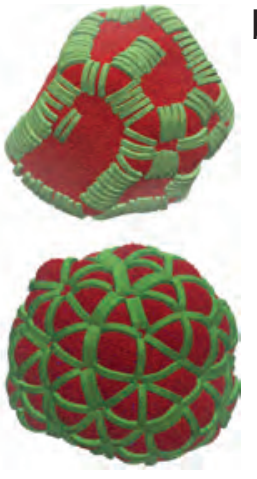
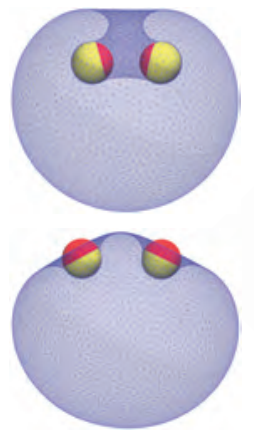

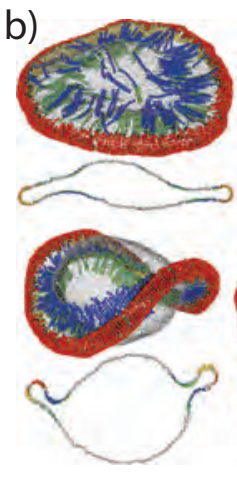

f)

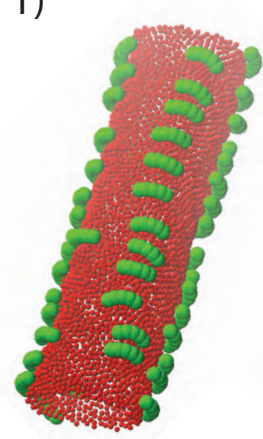

c)

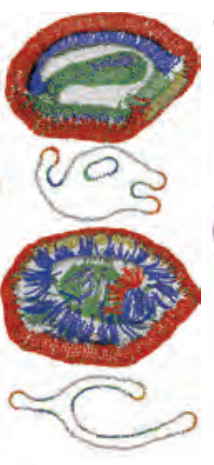

g)

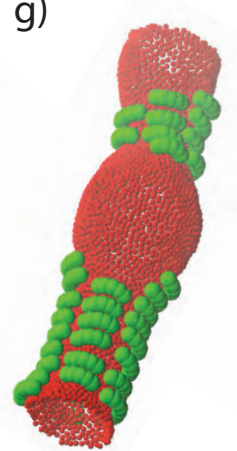

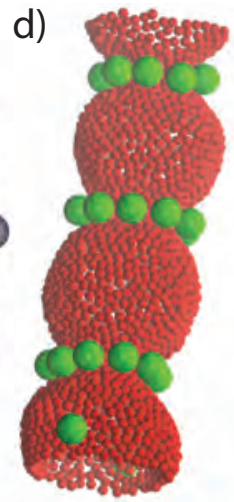

h)

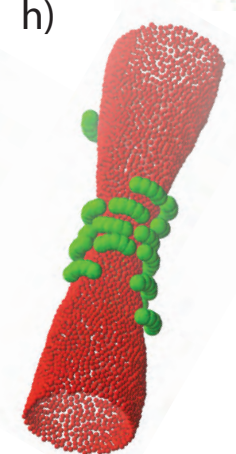

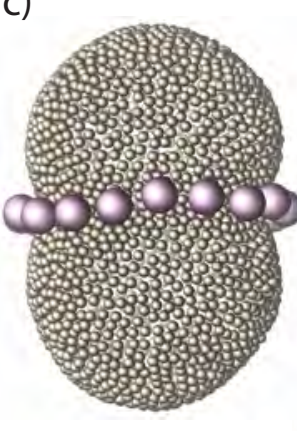

Figure 4: Examples of inclusion patterns formed on curved and closed membranes from recent simulation work. a) Chains and aster aggregates of curved rods at low (top row) and high (bottom row) adhesion, reproduced from Olinger et al. [56] with permission of The Royal Society of Chemistry. b) Deformed vesicle shape due to the combined action of oppositely curved rods at various rod-curvature ratios, reproduced from Noguchi and Fournier [57] with permission of The Royal Society of Chemistry. c) Spontaneous ring formation of colloids adhered to the outside of an elongated vesicle, from Vahid et al. [58], reproduced by permission of The Royal Society of Chemistry. d) Spontaneous ring formation of colloids adhered to the outside of a membrane tube. e) Interaction between two colloids partially adsorbed on the outside of a vesicle, for two different wrapping angles, adapted with permission from Bahrami and Weikl [59], copyright (2018) American Chemical Society. f-g) line and patch formation by curved inclusions with slightly different curvatures, adhered to the outside of a membrane tube. $\mathrm{h}$ ) Curvature-sensing by similar inclusions as in (f-g). d) and f-h) from Vahid et al. [55], Helle et al. [60] (CC BY 4.0).

Most effort has been put into modelling and measuring the interactions and assembly patterns between inclusions shaped like proteins known to interact with and induce membrane curvature, such as BAR domains and dynamin. Ramakrishnan et al. considered inclusions with mirror symmetry and found that they aggregate into ordered patches even at low concentrations and induce the membrane shape to change from spherical to disk-like and tubular [63]. Simunovic et al. found the formation of linear aggregates and meshes of N-BAR protein models and endocytic bud-like deformations of the membrane at higher protein densities [64]. An increase in surface tension decreases the interaction strength and range, and affects the preferred relative orientation and assembly patterns [65]. Describing protein scaffolds as elements of ellipsoidal or hyperboloidal surfaces, Schweitzer and Kozlov found that pairwise membrane-mediated interactions between strongly anisotropic scaffolds are always attractive with energies up to tens of $k_{\mathrm{B}} T$ [66]. Their computations showed that convex scaffolds tend to mutually orient to 
face the sides with the lowest contact angle.

Similar to the results of Simunovic et al. [64], Olinger et al. [56] found that bent rectangular rods that only adhere to the membrane with the concave side form chains at low adhesion strength and aster aggregates at higher adhesion strength (figure 4a). In both cases, the rods tend to line up parallel to each other at low adhesion strengths. Olinger et al. furthermore found that the aster arrangement originates from the saddlelike membrane deformations which induced a tilt of the lipids around the inclusions. On flat membranes, similar arrangements emerge, resulting in the formation of ridges, tubes, and buds, as shown by Noguchi [67]. However, mixing two types of bananashaped inclusions that induce opposite curvatures can inhibit the formation of tubes, instead leading to the formation of linear assemblies shaped like straight bumps and stripes on flat membranes and strongly deformed vesicles with rings of inclusions, as shown by Noguchi and Fournier [57] (figure 4b).

Experimental results on intrinsically curved proteins have shown that they can act as curvature sensors at low concentrations and curvature inducers at high concentrations [68, 69], leading to the formation of membrane tubes, consistent with the prediction of the simulations on both flat membranes and GUVs. Unsurprisingly, on membranes that are already tubular, banana-shaped inclusions will orient themselves along the bent direction. Recent work by Vahid et al. [55] shows that their interactions depend on the difference between their imposed curvature and that of the tube they reside on. Consequently, they may attract in a head-to-tail fashion forming rings, side-to-side fashion forming lines, or in both directions, forming scaffolds (figure $4 \mathrm{f}-\mathrm{g}$ ). The scaffolds themselves can change the tube's curvature considerably, leading to a phase-separation into regimes with high and low protein density, as directly observed in mitochondria by Helle et al. [60].

The recent simulation results, though already beautiful and thought-provoking, have likely only given us a first glance at what is to come in the near future. The coarsegrained model currently employed is likely to allow us to start exploring the wide range of membrane shapes found inside living cells, including the tubular and sheet-like networks in the endoplasmic reticulum, Golgi apparatus, and mitochondria, and dynamic processes like vesicle budding and cellular signalling. Key steps that will be taken in the coming years include the combination of different geometries into more complex shapes with different topologies, the further study of mixing and sorting of inclusions on those geometries, and the study of the conditions under which spontaneous changes in topology may occur. At the same time, there will be a drive towards more realistic models, mimicking more closely the properties of the various lipids and proteins found in living cells. From both approaches, basic principles will be extracted which in time will allow the design of membrane-with-inclusion systems that can self-organize into a functional shape.

\section{Conclusions}

Recent observations that proteins can sense and induce membrane curvature and use membrane-mediated interactions for organizing themselves into complex and functional structures has breathed new life into the search for a quantitative understanding of these interactions. Twenty-five years after the first attempt to formulate the interactions between two model inclusions on flat membranes, theorists have taken important steps 
towards refining and analytically describing how inclusions organize themselves on the biologically more relevant case of closed and curved membranes. The complexity encountered in membrane proteins such as many-body interactions, large deformations and arbitrary inclusion shapes remain challenging to analytical theories due to the inherent nonlinearities. Still, progress is being made steadily, and the first steps towards studying point-like inclusions on curved geometries have been taken, hopefully soon to be extended to further curved shapes.

The renewed interest in developing experimental model systems and the increasing sophistication of numerical methods opened the door to investigate situations that go beyond a few inclusions that weakly deform lipid membranes. The experimental model systems make use of model inclusions based on DNA-origami or colloidal particles on giant unilamellar vesicles. These model inclusions have the advantage of a tuneable membrane adhesion strength and controllable other interactions, and posses simple and uniform shapes, while allowing straightforward quantification of their membrane-mediated interactions through in situ techniques. The first measurements of the pairwise interaction potential of membrane-wrapped spheres on such model systems allowed a quantitative comparison with simulations for the first time. Easily accessible yet with far reaching insights are systematic measurements of multi-body interactions, from three-body interactions to collective effects. They would allow testing the non-additive nature of these interactions, probe the rich variety of phenomena predicted by simulations and provide quantitative insights into pattern formation in proteins.

Numerical simulations are leading the way in understanding and predicting the organization of model inclusions in lipid membranes. Modelling proteins with anisotropic shapes has provided valuable insights in recent years. However, the multi-body interactions between arbitrarily shaped inclusions or non-uniform composition still require significant work before a more general framework can be established. Biological membranes, such as the endoplasmic reticulum, are crowded with proteins and other molecules and feature complex, highly bent geometries. The most commonly employed descriptions to date do not suffice to describe such complex situations. We expect that the simulations will push towards this biologically relevant situation and provide profound insights on key processes such as cell division, trafficking and signalling.

\section{Acknowledgements}

We would like to thank the Netherlands Organisation for Scientific Research (NWO/OCW) and in particular the Frontiers of Nanoscience program, for funding two PhD positions that initiated our collaboration and contributed some of the work referenced here. We furthermore would like to thank all authors of the work highlighted in our figures for sharing their original image data with us.

\section{Funding}

This research did not receive any specific grant from funding agencies in the public, commercial, or not-for-profit sectors. 


\section{References with special $(*)$ / outstanding $(* *)$ interest}

* Šarić and Cacciuto [26] and Bahrami et al. [27] introduced coarse-grained methods now widely used in simulating colloid-membrane interactions.

* Grome et al. [38] demonstrated that vesicles can form tubules by self-assembling DNA nanosprings.

* Franquelim et al. [39] showed how and under what conditions DNA-origami inclusions modeled after BAR domains deform lipid vesicles.

* Sarfati and Dufresne [6] directly measured the interaction potential of two membraneadsorbed colloids both in the near and far field.

** van der Wel et al. [5] directly measured the interaction potential of two membraneadsorbed colloids, and showed that experimental results correspond directly with numerical predictions.

* Reynwar et al. [22]. First paper to show (through simulations) that interactions between many identical particles can lead to large membrane deformations and vesiculation.

* Agudo-Canalejo and Lipowsky [52]. Analytical work showing that adhered colloids can detect local curvature.

* Vahid and Idema [51]. Analytical study of interactions on tubular membranes, predicts the spontaneous formation of rings of isotropic inclusions.

* Olinger et al. [56]. Simulation study showing how curved bar-shaped inclusions can form ridges and networks on closed vesicles, with shapes depending on adhesion strength between inclusion and membrane.

* Noguchi and Fournier [57]. Numerical study of the effect of mixing two different kinds of curvature-inducing inclusions, resulting in, among others, highly deformed vesicles with large surface-to-volume ratios.

** Helle et al. [60]. Combined simulation and in vivo experimental work shows that bent proteins / inclusions can act as curvature sensors in low concentrations and curvature inducers in high concentrations, with patterns depending on the curvature of the inclusions. 


\section{References}

[1] B. Alberts, A. Johnson, J. Lewis, D. Morgan, M. Raff, K. Roberts, P. Walter, Molecular biology of the cell, 6th ed., Garland Science, New York, NY, U.S.A., 2014.

[2] M. Goulian, R. Bruinsma, P. Pincus, Long-range forces in heterogeneous fluid membranes, Europhys. Lett. 22 (1993) 145-150. URL: http://iopscience.iop.org/ 0295-5075/22/2/012. doi:doi:10.1209/0295-5075/22/2/012.

[3] H. T. McMahon, J. L. Gallop, Membrane curvature and mechanisms of dynamic cell membrane remodelling, Nature 438 (2005) 590-596. URL: http: //www . nature. com/articles/nature04396. doi:doi:10.1038/nature04396.

[4] M. Simunovic, P. Bassereau, G. A. Voth, Organizing membrane-curving proteins: the emerging dynamical picture, Curr. Opin. Struct. Biol. 51 (2018) 99-105. URL: http://linkinghub.elsevier.com/retrieve/pii/S0959440X1830023X. doi:doi: 10.1016/j.sbi.2018.03.018.

[5] C. van der Wel, A. Vahid, A. Šarić, T. Idema, D. Heinrich, D. J. Kraft, Lipid membrane-mediated attraction between curvature inducing objects, Sci. Rep. 6 (2016) 32825. URL: http://www. nature.com/articles/srep32825. doi:doi:10. 1038/srep32825. arXiv: 1603.04644.

[6] R. Sarfati, E. R. Dufresne, Long-range attraction of particles adhered to lipid vesicles, Phys. Rev. E 94 (2016) 012604. URL: http://link.aps.org/doi/10.1103/ PhysRevE.94.012604. doi:doi:10.1103/PhysRevE.94.012604. arXiv:1602.01802.

[7] N. Li, N. Sharifi-Mood, F. Tu, D. Lee, R. Radhakrishnan, T. Baumgart, K. J. Stebe, Curvature-driven migration of colloids on tense lipid bilayers, Langmuir 33 (2017) 600-610. URL: http://pubs.acs.org/doi/10.1021/acs.langmuir. 6b03406. doi:doi:10.1021/acs.langmuir.6b03406. arXiv:1602.07179.

[8] P. B. Canham, The minimum energy of bending as a possible explanation of the biconcave shape of the human red blood cell, J. Theor. Biol. 26 (1970) 61-81. URL: https://www.sciencedirect.com/science/article/pii/ S0022519370800327. doi:doi:10.1016/S0022-5193(70)80032-7.

[9] W. Helfrich, Elastic properties of lipid bilayers: theory and possible experiments, Zeitschrift für Naturforsch. C 28 (1973) 693-703. URL: http: //www.degruyter.com/view/j/znc.1973.28. issue-11-12/znc-1973-11-1209/ znc-1973-11-1209.xml. doi:doi:10.1515/znc-1973-11-1209.

[10] J.-M. Park, T. C. Lubensky, Interactions between membrane Inclusions on Fluctuating Membranes, J. Phys. I 6 (1996) 1217-1235. URL: http://www.edpsciences . org/10.1051/jp1:1996125. doi:doi:10.1051/jp1:1996125.

[11] K. S. Kim, J. Neu, G. Oster, Curvature-mediated interactions between membrane proteins, Biophys. J. 75 (1998) 2274-2291. URL: http://linkinghub.elsevier. com/retrieve/pii/S0006349598776726. doi:doi:10.1016/S0006-3495(98)77672-6. 
[12] C. Yolcu, R. C. Haussman, M. Deserno, The Effective Field Theory approach towards membrane-mediated interactions between particles, Adv. Colloid Interface Sci. 208 (2014) 89-109. URL: http://www. sciencedirect.com/science/article/ pii/S0001868614000748. doi:doi:10.1016/j.cis.2014.02.017.

[13] T. R. Weikl, M. M. Kozlov, W. Helfrich, Interaction of conical membrane inclusions: Effect of lateral tension, Phys. Rev. E 57 (1998) 6988-6995. URL: http: //link.aps.org/doi/10.1103/PhysRevE.57.6988. doi:doi:10.1103/PhysRevE.57. 6988. arXiv: 9804187.

[14] A. R. Evans, M. S. Turner, P. Sens, Interactions between proteins bound to biomembranes, Phys. Rev. E 67 (2003) 041907. URL: http://link .aps.org/doi/10.1103/ PhysRevE.67.041907. doi:doi:10.1103/PhysRevE.67.041907.

[15] P. Dommersnes, J.-B. Fournier, N-body study of anisotropic membrane inclusions: Membrane mediated interactions and ordered aggregation, Eur. Phys. J. B 12 (1999) 9-12. URL: http://link.springer.com/10.1007/s100510050968. doi:doi: $10.1007 / \mathrm{s} 100510050968$.

[16] S. Weitz, N. Destainville, Attractive asymmetric inclusions in elastic membranes under tension: cluster phases and membrane invaginations, Soft Matter 9 (2013) 7804. URL: http://xlink.rsc.org/?DOI=c3sm50954k. doi:doi:10.1039/ c3sm50954k. arXiv:arXiv:1307.7914v1.

[17] J. Guven, G. Huber, D. M. Valencia, Terasaki spiral ramps in the rough Endoplasmic Reticulum, Phys. Rev. Lett. 113 (2014) 188101. URL: http://link.aps.org/doi/ 10.1103/PhysRevLett.113.188101. doi:doi:10.1103/PhysRevLett.113.188101.

[18] M. Terasaki, T. Shemesh, N. Kasthuri, R. W. Klemm, R. Schalek, K. J. Hayworth, A. R. Hand, M. Yankova, G. Huber, J. W. Lichtman, T. A. Rapoport, M. M. Kozlov, Stacked endoplasmic reticulum sheets are connected by helicoidal membrane motifs, Cell 154 (2013) 285-296. URL: http://www.sciencedirect.com/science/ article/pii/S0092867413007708. doi:doi:10.1016/j.cell.2013.06.031.

[19] R. Capovilla, J. Guven, Stresses in lipid membranes, J. Phys. A: Math. Gen. 35 (2002) 302. URL: http://iopscience.iop.org/article/10.1088/0305-4470/ 35/30/302. doi:doi:10.1088/0305-4470/35/30/302.

[20] M. M. Müller, M. Deserno, J. Guven, Interface-mediated interactions between particles: A geometrical approach, Phys. Rev. E 72 (2005) 061407. URL: https:// link.aps.org/doi/10.1103/PhysRevE.72.061407. doi:doi:10.1103/PhysRevE.72. 061407. arXiv:0506019.

[21] C. Yolcu, M. Deserno, Membrane-mediated interactions between rigid inclusions: An effective field theory, Phys. Rev. E 86 (2012) 031906. URL: https://link.aps. org/doi/10.1103/PhysRevE.86.031906. doi:doi:10.1103/PhysRevE.86.031906. 
[22] B. J. Reynwar, G. Illya, V. A. Harmandaris, M. M. Müller, K. Kremer, M. Deserno, Aggregation and vesiculation of membrane proteins by curvature-mediated interactions, Nature 447 (2007) 461-464. URL: http://www. nature.com/doifinder/10. 1038/nature05840. doi:doi:10.1038/nature05840.

[23] S. J. Marrink, H. J. Risselada, S. Yefimov, D. P. Tieleman, A. H. de Vries, The MARTINI force field: Coarse grained model for biomolecular simulations, J. Phys. Chem. B 111 (2007) 7812-7824. URL: http://pubs.acs.org/doi/abs/10.1021/ jp071097f. doi:doi:10.1021/jp071097f. arXiv:NIHMS150003.

[24] L. Monticelli, S. K. Kandasamy, X. Periole, R. G. Larson, D. P. Tieleman, S.J. Marrink, The MARTINI coarse-grained force field: Extension to proteins, J. Chem. Theory Comput. 4 (2008) 819-834. URL: http://pubs.acs.org/doi/abs/ 10.1021/ct700324x. doi:doi:10.1021/ct700324x. arXiv:NIHMS150003.

[25] N. Ramakrishnan, P. B. Sunil Kumar, J. H. Ipsen, Monte Carlo simulations of fluid vesicles with in-plane orientational ordering, Phys. Rev. E 81 (2010) 041922. URL: http://link.aps.org/doi/10.1103/PhysRevE.81.041922. doi:doi:10.1103/ PhysRevE.81.041922.

[26] A. Šarić, A. Cacciuto, Fluid membranes can drive linear aggregation of adsorbed spherical nanoparticles, Phys. Rev. Lett. 108 (2012) 118101. URL: http://link.aps.org/doi/10.1103/PhysRevLett.108.118101. doi:doi:10.1103/ PhysRevLett.108.118101.

[27] A. H. Bahrami, R. Lipowsky, T. R. Weikl, Tubulation and aggregation of spherical nanoparticles adsorbed on vesicles, Phys. Rev. Lett. 109 (2012) 188102. URL: http://link.aps.org/doi/10.1103/PhysRevLett.109.188102. doi:doi:10. 1103/PhysRevLett.109.188102.

[28] K. K. Sreeja, J. H. Ipsen, P. B. Sunil Kumar, Monte Carlo simulations of fluid vesicles, J. Phys. Condens. Matter 27 (2015) 273104. URL: http://iopscience.iop. org/article/10.1088/0953-8984/27/27/273104. doi:doi:10.1088/0953-8984/27/ $27 / 273104$.

[29] R. A. Sauer, T. X. Duong, K. K. Mandadapu, D. J. Steigmann, A stabilized finite element formulation for liquid shells and its application to lipid bilayers, J. Comput. Phys. 330 (2017) 436-466. URL: https://linkinghub.elsevier.com/retrieve/ pii/S0021999116305836. doi:doi:10.1016/j.jcp.2016.11.004. arXiv:1601.03907.

[30] K. A. Brakke, Surface Evolver, Exp. Math. 1 (1992) 141-165. URL: http://www . jstor.org/stable/1575877?origin=crossref. doi:doi:10.2307/1575877.

[31] F. M. Ross, Opportunities and challenges in liquid cell electron microscopy, Science 350 (2015) aaa9886. URL: http://www.sciencemag.org/cgi/doi/10.1126/ science. aaa9886. doi:doi:10.1126/science.aaa9886.

[32] J. J. De Yoreo, S. N. A. J. M., Investigating materials formation with liquid-phase and cryogenic TEM, Nat. Rev. Mater. 1 (2016) 16035. URL: http://www.nature. com/articles/natrevmats201635. doi:doi:10.1038/natrevmats.2016.35. 
[33] D. Constantin, G. Brotons, A. Jarre, C. Li, T. Salditt, Interaction of Alamethicin pores in DMPC bilayers, Biophys. J. 92 (2007) 3978-3987. URL: http:// linkinghub.elsevier.com/retrieve/pii/S0006349507711979. doi:doi:10.1529/ biophysj.106.101204. arXiv:arXiv:1504.02883v1.

[34] F. Bories, D. Constantin, P. Galatola, J.-B. Fournier, Coupling between inclusions and membranes at the nanoscale, Phys. Rev. Lett. 120 (2018) 128104. URL: https://link.aps.org/doi/10.1103/PhysRevLett.120.128104. doi:doi:10.1103/ PhysRevLett.120.128104. arXiv:1803.10089.

[35] I. Koltover, J. O. Rädler, C. R. Safinya, Membrane mediated attraction and ordered aggregation of colloidal particles bound to giant phospholipid vesicles, Phys. Rev. Lett. 82 (1999) 1991-1994. URL: http://link.aps.org/doi/10.1103/ PhysRevLett.82.1991. doi:doi:10.1103/PhysRevLett.82.1991.

[36] A. Czogalla, D. J. Kauert, H. G. Franquelim, V. Uzunova, Y. Zhang, R. Seidel, P. Schwille, Amphipathic DNA origami nanoparticles to scaffold and deform lipid membrane vesicles, Angew. Chemie Int. Ed. 54 (2015) 65016505. URL: http://doi.wiley.com/10.1002/anie.201501173. doi:doi:10.1002/ anie.201501173. arXiv:arXiv:1408.1149.

[37] S. Kocabey, S. Kempter, J. List, Y. Xing, W. Bae, D. Schiffels, W. M. Shih, F. C. Simmel, T. Liedl, Membrane-assisted growth of DNA origami nanostructure arrays, ACS Nano 9 (2015) 3530-3539. URL: http://pubs.acs.org/doi/10.1021/ acsnano.5b00161. doi:doi:10.1021/acsnano.5b00161.

[38] M. W. Grome, Z. Zhang, F. Pincet, C. Lin, Vesicle tubulation with self-assembling DNA nanosprings, Angew. Chemie Int. Ed. 57 (2018) 5330-5334. URL: http:// doi.wiley.com/10.1002/anie.201800141. doi:doi:10.1002/anie.201800141.

[39] H. G. Franquelim, A. Khmelinskaia, J.-P. Sobczak, H. Dietz, P. Schwille, Membrane sculpting by curved DNA origami scaffolds, Nat. Commun. 9 (2018) 811. URL: http://www.nature.com/articles/s41467-018-03198-9. doi:doi:10.1038/ s41467-018-03198-9.

[40] C. Dietrich, M. Angelova, B. Pouligny, Adhesion of latex spheres to giant phospholipid vesicles: statics and dynamics, J. Phys. II 7 (1997) 1651-1682. URL: http: //www . edpsciences .org/10.1051/jp2:1997208. doi:doi:10.1051/jp2:1997208.

[41] A. Fery, S. Moya, P.-H. Puech, F. Brochard-Wyart, H. Mohwald, Interaction of polyelectrolyte coated beads with phospholipid vesicles, Comptes Rendus Phys. 4 (2003) 259-264. URL: http://linkinghub.elsevier.com/retrieve/pii/ S1631070503000306. doi:doi:10.1016/S1631-0705(03)00030-6.

[42] A. M. Mihut, A. P. Dabkowska, J. J. Crassous, P. Schurtenberger, T. Nylander, Tunable adsorption of soft colloids on model biomembranes, ACS Nano 7 (2013) 10752-10763. URL: http://pubs.acs.org/doi/10.1021/nn403892f. doi:doi:10. 1021/nn403892f. 
[43] C. van der Wel, N. Bossert, Q. J. Mank, M. G. T. Winter, D. Heinrich, D. J. Kraft, Surfactant-free colloidal particles with specific binding affinity, Langmuir 33 (2017) 9803-9810. URL: http://pubs.acs.org/doi/10.1021/acs.langmuir. 7b02065. doi:doi:10.1021/acs.langmuir.7b02065.

[44] C. van der Wel, D. Heinrich, D. J. Kraft, Microparticle assembly pathways on lipid membranes, Biophys. J. 113 (2017) 1037-1046. URL: http:// linkinghub. elsevier.com/retrieve/pii/S0006349517308445. doi:doi:10.1016/j. bpj.2017.07.019. arXiv:1612.03581.

[45] A. H. Bahrami, M. Raatz, J. Agudo-Canalejo, R. Michel, E. M. Curtis, C. K. Hall, M. Gradzielski, R. Lipowsky, T. R. Weikl, Wrapping of nanoparticles by membranes, Adv. Colloid Interface Sci. 208 (2014) 214-224. URL: http://www . sciencedirect. com/science/article/pii/S0001868614000694. doi:doi:10.1016/j.cis.2014.02.012.

[46] S. Dasgupta, T. Auth, G. Gompper, Nano- and microparticles at fluid and biological interfaces, J. Phys. Condens. Matter 29 (2017) 373003. URL: http://iopscience.iop.org/article/10.1088/1361-648X/aa7933. doi:doi: 10.1088/1361-648X/aa7933.

[47] M. Raatz, R. Lipowsky, T. R. Weikl, Cooperative wrapping of nanoparticles by membrane tubes, Soft Matter 10 (2014) 3570-3577. URL: http://xlink.rsc.org/ ?DOI=c3sm52498a. doi:doi:10.1039/c3sm52498a. arXiv:1401.1668.

[48] B. J. Reynwar, M. Deserno, Membrane-mediated interactions between circular particles in the strongly curved regime, Soft Matter 7 (2011) 8567. URL: http://xlink.rsc.org/?DOI=c1sm05358b. doi:doi:10.1039/c1sm05358b.

[49] A. B. Petrova, C. Herold, E. P. Petrov, Conformations and membrane-driven self-organization of rodlike fd virus particles on freestanding lipid membranes, Soft Matter 13 (2017) 7172-7187. URL: http://pubs.rsc.org/en/Content/ ArticleLanding/2017/SM/C7SM00829E. doi:doi:10.1039/C7SM00829E.

[50] T. Idema, S. Semrau, C. Storm, T. Schmidt, Membrane mediated sorting, Phys. Rev. Lett. 104 (2010) 198102. URL: https://link.aps.org/doi/10.1103/ PhysRevLett .104.198102. doi:doi:10.1103/PhysRevLett.104.198102.

[51] A. Vahid, T. Idema, Pointlike inclusion interactions in tubular membranes, Phys. Rev. Lett. 117 (2016) 138102. URL: http://link.aps.org/ doi/10.1103/PhysRevLett.117.138102. doi:doi:10.1103/PhysRevLett.117.138102. arXiv: 1510.03610.

[52] J. Agudo-Canalejo, R. Lipowsky, Uniform and Janus-like nanoparticles in contact with vesicles: energy landscapes and curvature-induced forces, Soft Matter 13 (2017) 2155-2173. URL: http://xlink.rsc.org/?DOI=C6SM02796B. doi:doi: 10.1039/C6SM02796B.

[53] R. Shlomovitz, N. S. Gov, Membrane-mediated interactions drive the condensation and coalescence of FtsZ rings, Phys. Biol. 6 (2009) 046017. URL: 
http://iopscience.iop.org/article/10.1088/1478-3975/6/4/046017. doi:doi: 10.1088/1478-3975/6/4/046017.

[54] P. G. Dommersnes, J. B. Fournier, P. Galatola, Long-range elastic forces between membrane inclusions in spherical vesicles, Europhys. Lett. 42 (1998) 233238. URL: http://iopscience.iop.org/article/10.1209/epl/i1998-00235-7. doi:doi:10.1209/epl/i1998-00235-7.

[55] A. Vahid, A. Šarić, T. Idema, Interactions between isotropic and banana-shaped inclusions on tubular membranes, unpublished (????).

[56] A. D. Olinger, E. J. Spangler, P. B. S. Kumar, M. Laradji, Membranemediated aggregation of anisotropically curved nanoparticles, Faraday Discuss. 186 (2016) 265-275. URL: http://xlink.rsc.org/?DOI=C5FD00144G. doi:doi:10.1039/ C5FD00144G.

[57] H. Noguchi, J.-B. Fournier, Membrane structure formation induced by two types of banana-shaped proteins, Soft Matter 13 (2017) 4099-4111. URL: http://xlink. rsc . org/?DOI=C7SM00305F. doi:doi:10.1039/C7SM00305F. arXiv:1702.04107.

[58] A. Vahid, A. Šarić, T. Idema, Curvature variation controls particle aggregation on fluid vesicles, Soft Matter 13 (2017) 4924-4930. URL: http://xlink.rsc.org/ ?DOI=C7SM00433H. doi:doi:10.1039/C7SM00433H.

[59] A. H. Bahrami, T. R. Weikl, Curvature-mediated assembly of Janus nanoparticles on membrane vesicles, Nano Lett. 18 (2018) 1259-1263. URL: http://pubs .acs . org/doi/10.1021/acs.nanolett.7b04855. doi:doi:10.1021/acs.nanolett.7b04855.

[60] S. C. J. Helle, Q. Feng, M. J. Aebersold, L. Hirt, R. R. Grüter, A. Vahid, A. Sirianni, S. Mostowy, J. G. Snedeker, A. Šarić, T. Idema, T. Zambelli, B. Kornmann, Mechanical force induces mitochondrial fission, eLife 6 (2017) e30292. URL: https://elifesciences .org/articles/30292. doi:doi:10.7554/eLife.30292.

[61] A. Šarić, A. Cacciuto, Mechanism of membrane tube formation induced by adhesive nanocomponents, Phys. Rev. Lett. 109 (2012) 188101. URL: http: //link.aps.org/ doi/10.1103/PhysRevLett.109.188101. doi:doi:10.1103/PhysRevLett.109.188101.

[62] M. Simunovic, G. A. Voth, A. Callan-Jones, P. Bassereau, When physics takes over: BAR proteins and membrane curvature, Trends Cell Biol. 25 (2015) 780-792. URL: http://www.sciencedirect.com/science/article/pii/ S0962892415001713. doi:doi:10.1016/j.tcb.2015.09.005.

[63] N. Ramakrishnan, P. B. Sunil Kumar, J. H. Ipsen, Membrane-mediated aggregation of curvature-inducing nematogens and membrane tubulation, Biophys. J. 104 (2013) 1018-1028. URL: http://linkinghub.elsevier. com/retrieve/pii/S0006349513000027. doi:doi:10.1016/j.bpj.2012.12.045. arXiv:arXiv:1302.1641v1. 
[64] M. Simunovic, A. Srivastava, G. A. Voth, Linear aggregation of proteins on the membrane as a prelude to membrane remodeling, Proc. Natl. Acad. Sci. USA 110 (2013) 20396-20401. URL: http://www.pnas.org/cgi/doi/10.1073/pnas.1309819110. doi:doi:10.1073/pnas.1309819110.

[65] M. Simunovic, G. A. Voth, Membrane tension controls the assembly of curvaturegenerating proteins, Nat. Commun. 6 (2015) 7219. URL: http://www nature.com/ doifinder/10.1038/ncomms8219. doi:doi:10.1038/ncomms8219.

[66] Y. Schweitzer, M. M. Kozlov, Membrane-mediated interaction between strongly anisotropic protein scaffolds, PLOS Comput. Biol. 11 (2015) e1004054. URL: http://dx.plos.org/10.1371/journal.pcbi.1004054. doi:doi: 10.1371/journal.pcbi.1004054.

[67] H. Noguchi, Acceleration and suppression of banana-shaped-protein-induced tubulation by addition of small membrane inclusions of isotropic spontaneous curvatures, Soft Matter 13 (2017) 7771-7779. URL: http://xlink.rsc .org/?DOI=C7SM01375B. doi:doi:10.1039/C7SM01375B. arXiv:1706.00185.

[68] B. Sorre, A. Callan-Jones, J. Manzi, B. Goud, J. Prost, P. Bassereau, A. Roux, Nature of curvature coupling of amphiphysin with membranes depends on its bound density, Proc. Natl. Acad. Sci. USA 109 (2012) 173-178. URL: http://www.pnas. org/cgi/doi/10.1073/pnas.1103594108. doi:doi:10.1073/pnas.1103594108.

[69] Z. Chen, E. Atefi, T. Baumgart, Membrane shape instability induced by protein crowding, Biophys. J. 111 (2016) 1823-1826. URL: http://dx.doi.org/10.1016/ j.bpj. 2016.09.039. doi:doi:10.1016/j.bpj.2016.09.039. 